\title{
Brownian dynamics of the self-assembly of complex nanostructures in the field of quasi-resonant laser radiation
}

\author{
Victoria S. Kornienko ${ }^{1,2}$, Aleksey S.Tsipotan ${ }^{1^{*}}$,Aleksandr S. Aleksandrovsky ${ }^{1,3}$ \\ and VitaliyV.Slabko ${ }^{1}$ \\ ${ }^{1}$ Siberian Federal University, Krasnoyarsk, Russia \\ ${ }^{2}$ Department of Computational Mathematics, Institute of Computational Modeling of Federal Research \\ Center KSC SB RAS, Krasnoyarsk, Russia \\ ${ }^{3}$ Laboratory of Coherent Optics, Kirensky Institute of Physics Federal Research Center KSC SB RAS, \\ Krasnoyarsk, Russia \\ Corresponding author. \\ E-mail addresses: acipotan@sfu-kras.ru (Aleksey S.Tsipotan)
}

\begin{abstract}
Self-assembly of nanoparticles under the action of laser field can be an universal method for the formation of nanostructures with specific properties for application in sensorics and nanophotonics. For prognosis of the self-assembly processes, the model of movement of an ensemble of nanoparticles in a viscous media under the action of laser radiation with the account for interaction of laser-induced polarizations and Brownian dynamics is developed. This model is applied to the investigation of the self-assembly process of a triple of nanoparticles into three-particle structure with a predetermined geometry.Two specific cases of formation of nanostructure from a preliminarily formed pair of particles are studied: either for the pair fixed in space or from the unfixed pair of nanoparticles. The geometry of resulting nanostructures is shown to be determined by the polarization direction of laser radiation and the laser wavelength. Under proper choice of these parameters the formation of structures is shown to be highly efficient. E. g., maximum probability of structures formation is as hig as $36-46 \%$ per single laser pulse of $10 \mathrm{~ns}$ duration.
\end{abstract}

Keywords: dipole-dipole interaction, laser field, Brownian dynamics, self-assembly of nanostructures, colloidal crystals

\section{Introduction}

Studies in the area of nanotechnology presently exhibit extremely fast rate of development. As an example, the applications of new nanomaterials are under intense research in the materials science [1]. Another prospective subject of studies are nanoliquids containing small amounts of nanoparticles in, e.g., water solutions [2]. Consequently, great attention of researchers over last decades is paid to the production [3,4], characterization of properties [5-8], and applications of new types of nanoparticles and structures based on them [9].

Obtaining of the nanostructures with unique properties differing from those of the bulk material and depending both on the composition and shape becomes more and more actual. Therefore, the problem of development of universal technique of nanostructures' formation sharply stands on the agenda of many researchers.

One of approaches used for nanostructures formation is based on the molecular processes of self-assembly of nanoparticles. Such self-assembly is performed with the help of modification of the surface of nanoparticles by molecules of DNS [10,11], peptides, lipids and polymers[12], the selective interaction of which determines the geometry of the formed structure. 
However, such an additional modification of the nanoparticles' surface may lead to the changes in their properties. To avoid this, the alternative approach can be used that is based on the application of physical interactions, particularly, hydrothermal processing [13], convective flows, magnetic field or illumination by the light [14]. For instance, large-area golden nanostructures were fabricated via annealing of the samples containing golden nanoparticles under different temperatures [15]. Laser ablation ov various materials by ultrashort pulses was used for obtaining the periodic structures on a surface [16]. The illumination by the light also can be used for optical binding of nanoparticles via either near-field or farfield interactions [17], that leads to the formation of structures with the dimensions either smaller or of the order of the external radiation wavelength, correspondingly. Presence of optical resonances in nanoparticles leads to substantial peculiarities both in the process of nanostructures' formation in the field of laser radiation and in the peculiarities of their optical spectra [18]. Particularly, the authors of [19] have theoretically shown that the action of resonant laser radiation onto the ensembles of silver nanoparticles possessing plasmon resonances leads to, for the first, enhancement of near-field interaction between particles, and, for the second, gives the possibility of the control of the geometry of nanostructure forming via induced self-assembly by the tuning of the laser wavelength. However, low Q-factor of plasmonic resonances prevents achieving the interparticle interaction energy that would exceed the thermal movement energy $(\mathrm{kT})$ at intensities of laser radiation below the threshold of radiative stiffness if silver nanoparticles. Further, in [20] it was shown that choosing the colloidal quantum dots that possess better Q-factor resonances allows obtaining enough interparticle interaction energy in the field specified above. Molecular dynamics modeling of the self-assembly of quantum dots into a stable pair [21] and subsequent experiment [22] with the parameters of the medium and the field obtained from the modeling have shown the possibility of formation of stable structure consisting of two CdTe quantum dots. However, of grater interest is the possibility of forming the structures consisting of three and more particles, with the properties of structures being controllable via changing the geometry of a structure. We also note that analytical calculation of the energy of dipole-dipole interaction does not allow estimation of the probability of aggregation and choosing the optimum parameters of the experiment, since the potential well depth and spectral position are in dependence on the interparticle distances and mutual positions of paricles with respect to polarization plane of laser radiation. The influence of particle movement on the probability of structure formation does not allow quantitative preliminary analysis and must be accounted in the process of numerical modeling.

Present paper proposes the approach to the formation of complex nanostructures from initially isolated nanoparticles under the action of laser radiation. A step-by-step aggregation is considered when a third particle adjoins to a preliminarily formed pair of particles, the position of the former being controlled by the choice of the wavelength and the polarization of the external field. Within this case, two variants can be implemented, namely, either initially formed pair can be fixed in space or be arbitrarily oriented with respect to laser polarization.

We suggest the dynamical model of the laser-induced self-assembly of nanostructures based on the molecular dynamics in the field of laser radiation, and develops it to investigate the possibility of assembly of three-particle nanostructures with the demonstration of the shape control. For the modeling of the processes of both spontaneous and laser induced aggregation, the description of a system with the help of the Langevin equations system [6] is used, with the account for Brownian dynamics of the movement of an ensemble of nanoparticles. Numerical solution of the equations system was implemented according to the explicit two-stage Runge-Kutta scheme. Conducting large enough runs on the scheme, we statistically calculate the probability of formation of either pyramidal, or linear, or rectangular structures, in dependence on initial particles positions and the parameters of external field.

\section{Theory and model}

The most facile and the least expensive method of obtaining the colloid crystals, i.e. highly ordered structures of nanoparticles that does not require any local physical action onto the system is based on the ability of nanoparticles to selforganize in the process of random Brownian collisions in real disperse systems [23]. However, when using this approach, it is impossible to control the processes of formation of pre-defined nanostructures. The method of formation of nanostructures with a pre-defined geometry from the nanoparticles possessing resonances in the optical wavelength range under the action of laser radiation was proposed in $[19,20]$. The essence of the method is as follows. Illumination of microobjects by laser radiation induces the polarization on them that is the cause of interaction of the particle both with the external field, allowing the formation of the structures with the dimensions of the order of the wavelength, and with other particles. In the latter case the formation of structures with the dimensions much smaller than the wavelength of laser radiation inducing the polarization is possible. Presence of optical resonances leads to the enhancement of interparticle interaction and is the base for selective formation of various structures with the pre-defined positions of particles within the structure, for the sake of the dependence of interaction energy between particles illuminated by laser radiation on the laser frequency, on the resonant frequencies of 
particles and on the orientation of groups of particles with respect to polarization plane of radiation. In this case, a potential well is formed in the spectral and angular dependences of the interparticle interaction energy that corresponds to the given configuration of the group. If the depth of this potential well is larger than the energy of a barrier preventing from spontaneous aggregation [21], then self-organized merging of the group under consideration into a nanostructure with predefined configuration becomes possible, the configuration being determined by two independent parameters, namely, laser frequency and laser polarization orientation. After the laser switch-off such structure is preserved stable due to the Van der Waals interaction.

\subsection{Mathematical basis}

For the description of the self-organization of particles in the field of laser radiation we will use the model outlined in $[19,20]$. Let us consider the ensemble of $N$ particles with the masses $m_{j}$, radii $R_{j}$ and resonant frequencies $\omega_{j}^{0}$ $(j=1, \ldots, N)$. The particles are illuminated by laser radiation, and they interact with each other due to their dipole moments induced by the external light field. For every $j$-th dipolar particle in every time moment we define following quantities: $\stackrel{\mathrm{r}}{r}_{j}=\left(r_{j}^{x}, r_{j}^{y}, r_{j}^{z}\right)^{T}$ is the radius vector of the center of mass of a dipole particle;

$\stackrel{\mathrm{r}}{V_{j}}=\left(V_{j}^{x}, V_{j}^{y}, V_{j}^{z}\right)^{T}$ is the vector of translational velocity of the center of mass; $\stackrel{\mathrm{r}}{d_{j}}=\left(d_{j}^{x}, d_{j}^{y}, d_{j}^{z}\right)^{T}$ is the vector of induced dipole moment.

In the course of modeling the probability was determined of convergence of particles to the distance between their centers of mass $\max \left|r_{j k}^{\mathrm{r}}\right| \leq 2 R_{j}$ during $T \leq 10 \mathrm{~ns}$, at various orientations of particles with respect to polarization plane of laser radiation and its frequency. Pulse duration quoted above was chosen to be equal to the duration of laser pulse used in the experiment on the structures formation [21].

According to [17], the field propagator between field-induced dipole moments depends on the distance and can be written as the sum of three terms each of them being dominating at certain distance range.

$$
\begin{array}{r}
G_{i j}=\frac{\exp \left(i k\left|r_{j k}\right|\right)}{4 \pi \varepsilon_{0} \varepsilon\left|r_{j k}\right|^{3}}\left[\left(3-3 i k\left|r_{j k}\right|-k^{2}\left|r_{j k}\right|^{2}\right) \frac{\left|r_{k}\right|\left|r_{j}\right|}{\text { umur }^{2}}\right. \\
\left.+\left(k^{2}\left|r_{j k}\right|^{2}+i k\left|r_{j k}\right|-1\right) \delta_{j k}\right],
\end{array}
$$

where $k=2 \pi / \lambda$ is the wave number of the light in the medium, $\varepsilon_{0}$ is the permittivity of vacuum, $\varepsilon$ is the relative permittivity of the medium, and $\delta_{j k}$ denotes the Kronecker delta. The term responsible for near-field interaction $\left(\left|r_{j k}\right|^{-3}\right)$ is the largest at interparticle distances $\left|r_{j k}^{\mathrm{r}}\right|<<\lambda$ (where $\lambda$ is the wavelength of external field) and allows formation of the structures with the dimensions much smaller than the wavelength. Two other terms considerably contribute to the interaction energy at the distances of order of wavelength $\left(\left|r_{j k}^{\mathrm{r}}\right|^{-2}\right)$ or at the distances $\left|r_{j k}^{\mathrm{r}}\right|>>\lambda\left(\left|r_{j k}^{\mathrm{r}}\right|^{-1}\right)$ and can be responsible for the formation of structures with dimensions equal or exceeding the external field wavelength. Note that the latter structures exist only in the presence of standing wave of external field. Structures formed in the near field can exist in the absence of the field due to van der Waals force [21]. In the present study, we will assume that the dimensions of the whole ensemble are much smaller than the wavelength of incident radiation. This assumption allows neglecting the phase shift between induced dipole moments and considering the field strength of external light field $\stackrel{\mathrm{I}}{E}$ to be homogeneous over the ensemble and to avoid taking the retardation into account. Then the electric field strength vector of the light wave field is representable in the form of harmonic function dependent only on time with the oscillation frequency $\omega^{r}, \stackrel{1}{E}=1 / 2 E_{0} \exp \left(i \omega^{r} t\right)+c . c$.

When considering a local field in the vicinity of $j$-th particle, both contribution of external field and those from all the rest particles must be taken into account via summing of the fields of all dipoles induced on them. Then the dipole moment vector induced on $j$-th particle has the form 


$$
\stackrel{\mathrm{r}}{d}=\chi_{0 j}\left(\stackrel{\mathrm{r}}{E}+\sum_{k \neq j} \stackrel{\mathrm{r}}{E_{k}}\right)
$$

where $\chi_{0 j}$ is the linear polarizability of isolated $j$-th particle, $\stackrel{1}{E_{k}}$ is the field created by $k$-th particle of ensemble in the point of location of $j$-th particle:

$$
\stackrel{\mathrm{r}}{E_{k}}=\frac{1}{4 \pi \varepsilon_{0}} \frac{3\left(\stackrel{\mathrm{r}}{d_{k}}, \underset{r}{r} r_{j k}\right) \underset{r_{j k}}{\mathrm{r}}-\stackrel{\mathrm{r}}{d_{k}}\left|r_{j k}\right|^{2}}{\left|r_{j k}\right|^{5}},
$$

where $\stackrel{1}{r}_{j k}=\stackrel{1}{r}_{j}-\stackrel{1}{r}_{k}, \varepsilon_{0}=(36 \pi)^{-1} \cdot 10^{-9} \mathrm{C}^{2} /\left(\mathrm{N} \cdot m^{2}\right)$ is the dielectric permittivity of vacuum.

According to [24] and using (2), we obtain the expression describing the energy of $j$ - th dipole:

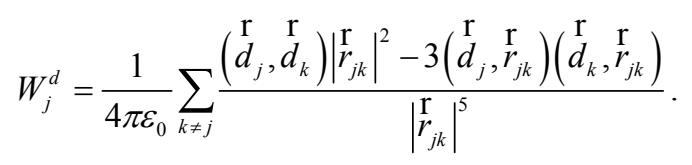

For microscopic description of dipolar polarizability of individual particle we will use two-level model [19, 20]. In the frame of this model under simplest case of solitary resonance at the frequency $\omega^{r}$ characterized by electric dipole moment of the transition $\left|d_{12}\right|^{2}$ and excitation relaxation rate (homogeneous width of resonance) $\Gamma$, dipole polarizability of particle in the vicinity of $\omega^{r}$ can be described by the following expression:

$$
\chi_{0}=\frac{\left|d_{12}\right|^{2}}{\mathrm{~h}(\Omega+i \Gamma)},
$$

where $\Omega=\omega^{r}-\omega$ is the detuning from resonance, and $\mathrm{h}$ is the Planck constant.

\subsection{Equations of motion}

Translational motion of $j$-th particle of the ensemble is described by the Langevin equations system

$$
\left\{\begin{array}{l}
d r_{j} / d t=\stackrel{\mathrm{r}}{v_{j}}, \\
m_{j}\left(d{\left.\stackrel{\mathrm{r}}{v_{j}} / d t\right)}^{\mathrm{r}}=F_{j}-\underset{\mathrm{r}}{F_{j}^{f}}+\stackrel{\mathrm{r}}{F_{j}^{c}},\right.
\end{array}\right.
$$

where $\stackrel{1}{F}_{j}=-\operatorname{grad}\left(W_{j}^{v}+W_{j}^{e}+W_{j}^{d}\right), \stackrel{1}{F}_{j}^{f}$ is the viscous friction force, $\stackrel{1}{F}_{j}^{c}$ is the stochastic hydrodynamic force.

Pair interaction energy includes the energy of Van der Waals interaction and that of electrostatic Coulomb repulsion responsible for the protection of particles against spontaneous aggregation. For the description of van der Waals interaction we use following expression [23]:

$$
\begin{aligned}
& W_{j}^{v}=-\frac{A_{H}}{6} \sum_{k \neq j}\left(\frac{2 R_{j}^{2}}{h_{j k}^{2}+4 R_{j} h_{j k}}+\frac{2 R_{j}^{2}}{h_{j k}^{2}+4 R_{j} h_{j k}+2 R_{j}^{2}}+\right. \\
& \left.+\ln \frac{h_{j k}^{2}+4 R_{j} h_{j k}}{h_{j k}^{2}+4 R_{j} h_{j k}+2 R_{j}^{2}}\right)
\end{aligned}
$$

where $A_{H}$ is the effective Hamaker constant, $R_{j}$ is the radius of particle, $h_{j k}=\left|r_{j k}^{\mathrm{r}}\right|-R_{j}-R_{k}$ is the interparticle gap. 
Potential energy of Coulomb interaction between overlapping double electric layers $W_{j}^{e}$ for $j$-th spherical particle with radius $R_{j}$ is given by the known expression [23]:

$$
W_{j}^{e}=2 \pi \varepsilon \varepsilon_{0} R_{j} \phi_{0}^{2} \sum_{k \neq j} \ln \left[1+\exp \left(-h_{j k} k_{0}\right)\right]
$$

where $k_{0}=1 / \lambda_{0}$ is the shielding constant (here $\lambda_{0}$ is the Debye-Huckel radius), $\varepsilon$ is the dielectric permittivity of the environment, $\phi_{0}$ is the potential at the boundary of Helmholtz layer.

It is assumed that the medium parameters are chosen in such a way that electrostatic repulsion force exceeds Van der Waals force but action of laser radiation could lead to the aggregation of particles into structures.

Viscous friction force being the main factor of kinetic energy dissipation, for spherical particles with the radius $R_{j}$ is determined by the Stokes formula

$$
\stackrel{\stackrel{1}{F}}{f}=6 \pi \eta R_{j} \stackrel{\mathrm{r}}{v}_{j}
$$

here $\eta$ is the dynamic viscosity of the medium.

Interaction of particles with the environment with fluctuating density leads to random change of the trajectory of their motion that must compensate for the friction force effect and to preserve dispersed phase mobility. To account for Brownian motion, we assume that random force $\stackrel{1}{F}_{j}^{c}$ is featured by Gaussian distribution. During the temporal step $\Delta t$ the particle steadily undergoes the random force action. Before each step of integration the values of projections of random force onto coordinates' axes $\left(\stackrel{\mathrm{r}}{F_{i}^{c}}\right)^{k}(k=x, y, z)$ is chosen from Gaussian distribution with the zero average value and standard deviation

$$
\delta^{2}=\frac{12 \pi \eta R_{j} k_{b} T}{\Delta t}
$$

here $k_{b}$ is Boltzmann constant and $T$ is the temperature of the medium.

Account for all forces acting onto the particles in the field of quasiresonant radiation allows modeling of the process in question with the help of Brownian dynamics and estimation of necessary parameters of the medium and the field. In the general case, the integration of the equation system (7) can be done only numerically.

\subsection{Verification of the model}

According to estimates quoted in [16-19], let us choose following parameters as the starting data:

$$
\begin{gathered}
T=300 \mathrm{~K}, \eta=0.8902 \mathrm{mPa} \cdot \mathrm{sec}, r_{i}=1.5 \mathrm{~nm},\left|d_{12}\right|^{2}=1.91 \cdot 10^{-44} \mathrm{~J} \cdot m^{3}, \lambda_{j}^{0}=525 \mathrm{~nm}, \lambda_{0}=9.8 \mathrm{~nm}, \varphi_{\delta}=2.34 \mathrm{mV}, \\
\Delta \lambda=3 \mathrm{~nm}, m_{i}=2.12 \cdot 10^{-23} \mathrm{~kg}, \quad A_{H}=50 k_{b} T .
\end{gathered}
$$

We define intensity of laser field equal to $I=10^{6} \mathrm{~W} / \mathrm{cm}^{2}$. It is necessary to note that in real Brownian system, the velocities of particles obey Maxwell distribution [25, 26]; however, since the formation of thee-particle structure is performed step by step then we set translational velocity at starting time moment $t=t_{0}$ to be zero that corresponds to mathematical expectation of supposed distribution. Minimal allowed distance between centers of mass of dipole particles $d_{\text {min }}$ is taken to be double radius of the particles $d_{\text {min }}=2 R_{j}$.

As it was already pointed, for seeking of numerical solution of ODE system (5), the second order method from the family of explicit numerical Runge-Kutta methods was used [27].

Calculation of particles' motion was conducted in a spherical cell with initial number of particles distributed within it being equal to $N$. The radius of the cell was equal to $20 \mathrm{~nm}$. At every iteration step for every particle, independently on either the particle is isolated or involved into an aggregate, the motion equations (5) were solved in accordance with model parameters described above. Numerical method was implemented at the uniform grid with the step $h=0.01 \mathrm{~ns}$. Random force 
$\stackrel{\mathrm{r}}{F_{j}^{c}}$, as pointed in 2.3, is governed by Gaussian distribution. Numerical implementation of random force was done using random number generator that produced random uniformly distributed quantity further converted via Boxer-Mueller transformation to Gauss distribution in accordance with parameters defined in 2.3. Probability of assembly of particles into a structure was evaluated from the set containing 100 calculation runs of the same type. Deviation from the mean value did not exceeded $10 \%$.

\section{Results and discussion}

As we pointed above, formation of complex structures can be performed in the course of step-by-step assembly when a third particle adjoins to preliminarily formed pair at desired angle. Geometry of formed structure can be controlled by the frequency and orientation of polarization vector of external field. In this case initial pair can be either fixed, e.g., with additional laser field, or unfixed, with the respect to polarization plane. In the course of modeling special attention was paid to monitoring the formation of stable structures: «line», when third particle adjoins the pair at the angle $0^{\circ} \pm 5^{\circ}$ from line connecting centers of mass of particles composing the pair, «angle» $\left(90^{\circ} \pm 5^{\circ}\right)$, «pyramid» $\left(120^{\circ} \pm 5^{\circ}\right)$.

\subsection{Formation of structure from three particles with spatially oriented pairs of particles}

Let us consider the case when particles 1 and 2 (Fig. 1) are fixed and their orientation in space with respect to the polarization plane of inducing field is enabled by additional light field. The task is to calculate numerically the energy of interaction between the third particle and particles 1 and 2 in dependence on the wavelength and orientation of polarization vector of external field, that would provide values of these parameters that enable formation of potential well with maximum depth. Positions of particles in the initial moment of time are presented in Fig. 1. Distance between particles within the pair is chosen to be $\left|r_{12}^{\mathrm{r}}\right|=R_{1}+R_{2}$, while the distance between second and the third particles $\left|r_{23}^{\mathrm{r}}\right|$ was set to be 20,16 or $10 \mathrm{~nm}$; the angle $\theta$ between $\stackrel{\mathrm{r}}{r}_{12}$ and $\stackrel{\mathrm{r}}{r}_{23}$ can be arbitrary. Preliminary estimates show that chosen distance corresponds to the interparticle interaction energy larger than the thermal motion energy $(\mathrm{kT})$.

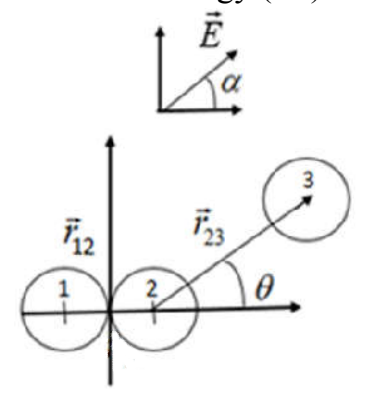

Fig. 1. Initial position of particles.

The calculated dependence of dipole-dipole interaction energy (3) normalized to the thermal energy $k T$ at room temperature, for the case of three nanoparticles with spatially oriented pair, on the wavelength of external field and on the angle $\alpha$ between polarization vector and the line connecting particles 1 and 2 is plotted in Fig. $2,{ }_{23}^{r}=16 \mathrm{~nm}: \theta=0^{\circ}$ (Fig. 2a), $\theta=120^{\circ}$ (Fig. 2b). 
a)

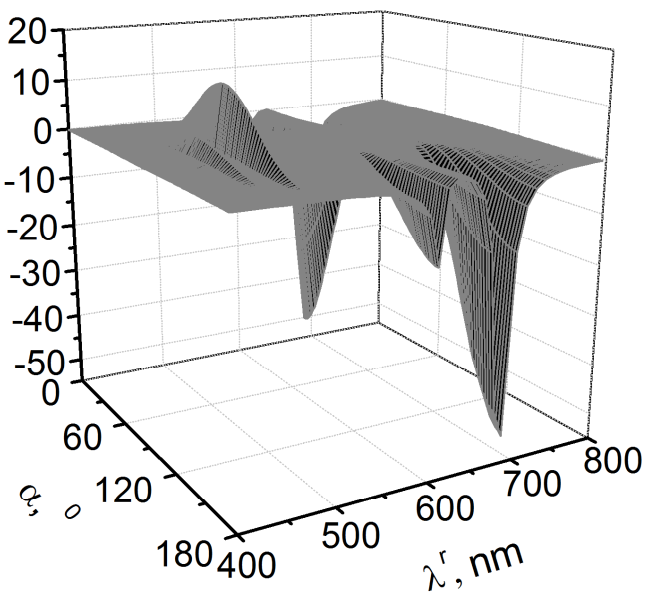

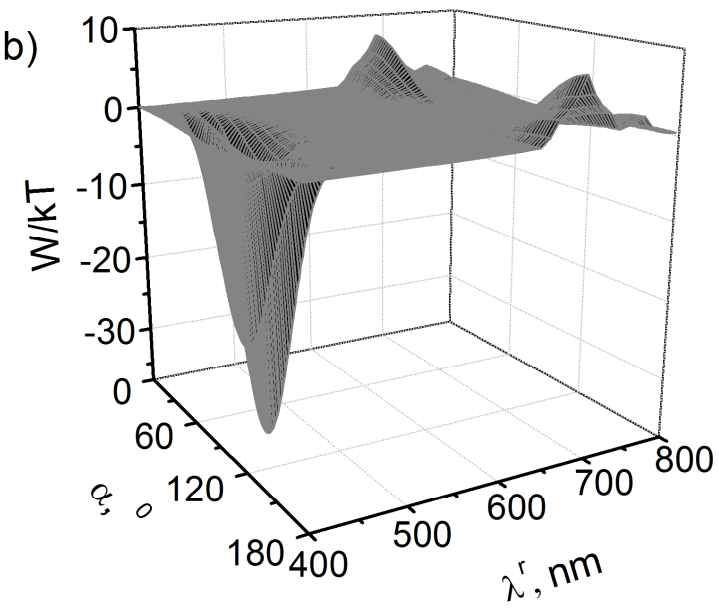

Fig. 2. Dependence of dipole-dipole interaction energy of three nanoparticles including spatially fixed pair after averaging over time, on the angle $\alpha$ and on the wavelength of external field $\lambda^{r} ; \stackrel{1}{r}_{23}=16 \mathrm{~nm}, \theta=0^{\circ}$ (2a), $\theta=120^{\circ}(2 \mathrm{~b})$.

As one can see from Fig. 2, the choice of the angle $\alpha$ defining the direction of polarization vector of external field as well as the choice of wavelength and position of the third particle significantly influence the behavior of energy dependence. For $\theta=0^{\circ}$ at $\alpha=0^{\circ}$ and at $\alpha=180^{\circ}$ (Fig. 2a) the potential well in the interparticle interaction energy is observed at $690 \mathrm{~nm}$ with the depth of order $-50 \mathrm{kT}$, evidencing the possibility to produce stable nanostructure. Spectral width of the well is of order of $200 \mathrm{~nm}$. Different behavior is observed for $\theta=120^{\circ}$ with the potential well as deep as $-33 k T$ is attained at $\alpha=90^{\circ}$ (Fig. 2b). In this case potential well experiences short wavelength shift and corresponds to the wavelength $450 \mathrm{~nm}$.

Reducing the distance between fixed pair and the third free particle leads to increase of detuning from the resonance, and the resonance becomes shifted to the longer-wavelength (Fig. 3a) or shorter-wavelength regions of spectrum (Fig. 3b) for the first and second cases, correspondingly.

Therefore, wavelengths $\lambda^{r}=690 \mathrm{~nm}$ and $\lambda^{r}=450 \mathrm{~nm}$ correspond to the maximum attractive interaction energy and can be chosen for the formation of a nanostructure on the base of spatially fixed pair of particles. There was assumed in the calculations that the third particle was located on a circle centered in particle 2 with the radius $16 \mathrm{~nm}$ that corresponds to volume concentration $n=0.0025$. For the wavelength of external field $\lambda^{r}=690 \mathrm{~nm}$ the calculated probability of structure formation from three particles during time period not more than $10 \mathrm{~ns}$ is presented in Fig. 4.

Calculation shows that for $\lambda^{r}=690 \mathrm{~nm}$ maximum probability of $46 \%$ is attained for linear structure with average formation time $6.81 \mathrm{~ns}$. The angle of external field polarization direction corresponding to maximum probability equals to $0^{\circ}$, in accordance with dipole-dipole interaction energy calculation (Fig. 2a). In 29\% of runs the adjoining does not take place and in $25 \%$ of runs the adjoining leads to the formation of structures other than the linear one. Similar calculations for the wavelength of the external field $\lambda^{r}=450 \mathrm{~nm}$ are shown in Fig. 5.

In this case the structures of pyramid-type and angle-type are formed with the probabilities $39 \%$ and $24 \%$ correspondingly, and in $37 \%$ of runs the adjoining does not take place. Average aggregation time into pyramid-type structure is $4.49 \mathrm{~ns}$, while for angle-type structure it is $5.76 \mathrm{~ns}$. The angles of external field polarization direction corresponding to maximum adjoining probability are equal to $45^{\circ}$ for angle-type and $90^{\circ}$ for pyramid-type structures and correspond to the calculated ones (Fig. 2b).

Therefore, for fixed pair of particles at external field wavelength $690 \mathrm{~nm}$ the formation of linear structure is the most probable, and the most favorable value of $\alpha=0^{\circ}$. For external field wavelength $450 \mathrm{~nm}$ the most probable structure is the pyramid, and the most favorable value of $\alpha=90^{\circ}$.

Calculation show that under the choice of the wavelength and external field vector direction with respect to fixed pair of particles the production of three different stable structures is possible, namely, linear-type and pyramid-type ones and angle-type. Structure formation probability may reach $46 \%$ per laser pulse at modest external field intensity. 

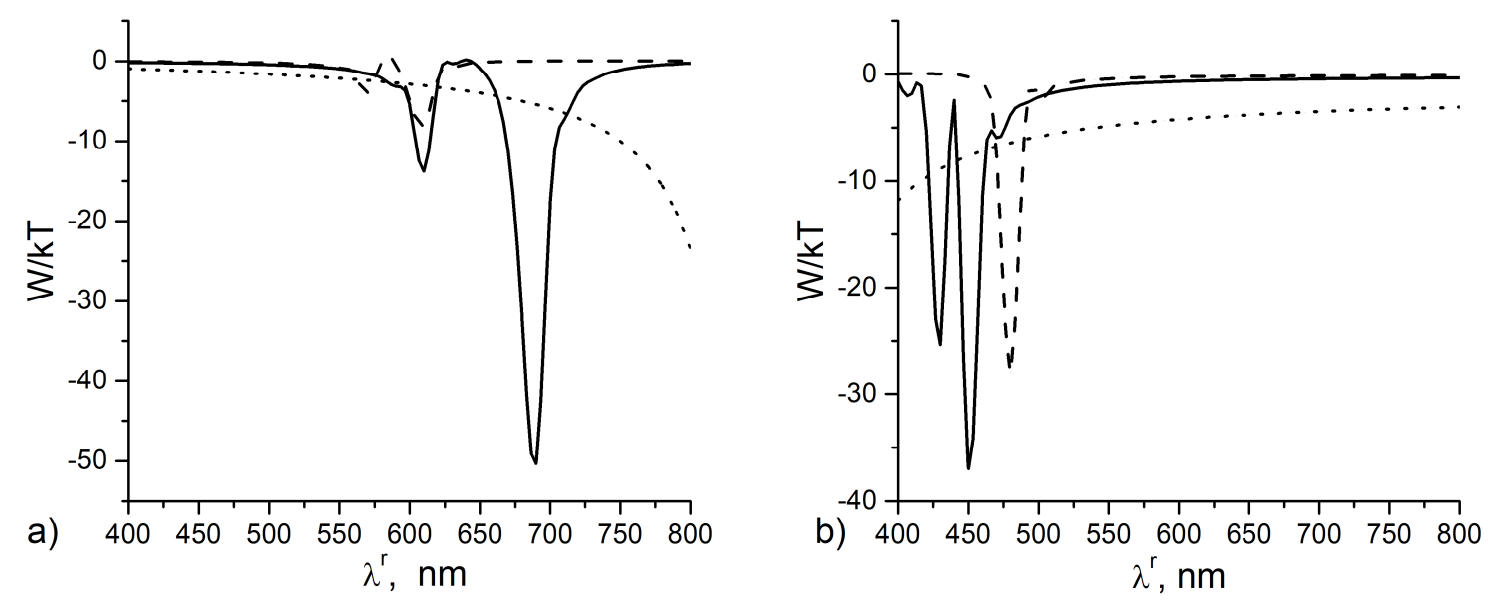

Fig. 3. Time-averaged dipole-dipole interaction energy of three particles with spatially oriented pair in dependence on the interparticle distance at $\theta=0^{\circ}, \alpha=0^{\circ}$ (За) и $\theta=120^{\circ}, \alpha=90^{\circ}(3 \mathrm{~b})$. Dash lines correspond to interparticle distance $r_{23}=20 \mathrm{~nm}$, solid lines $16 \mathrm{~nm}$, dot lines $-10 \mathrm{~nm}$.

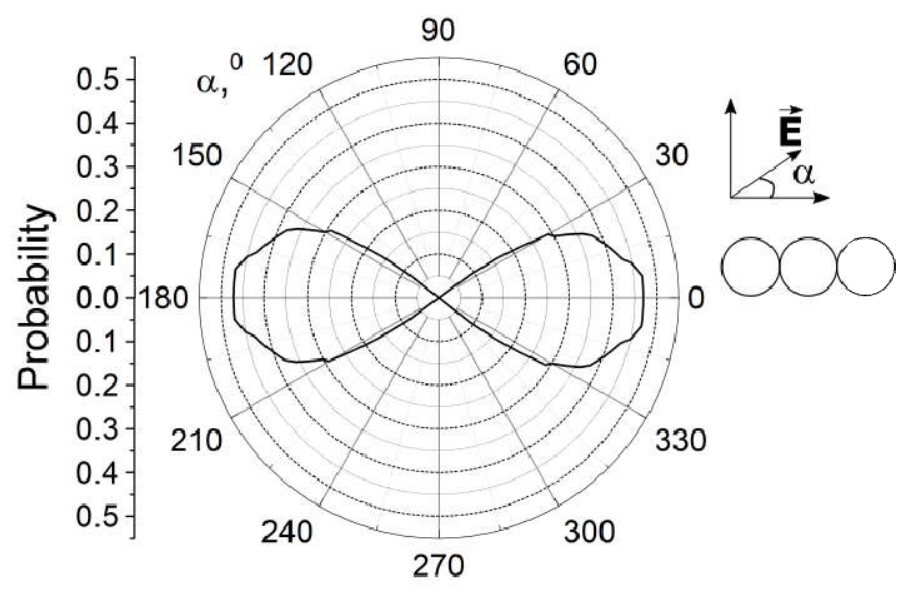

Fig. 3. Probability of formation of linear structure from three particles with spatially oriented pair in dependence on their mutual orientation at $\lambda^{r}=690 \mathrm{~nm}$.

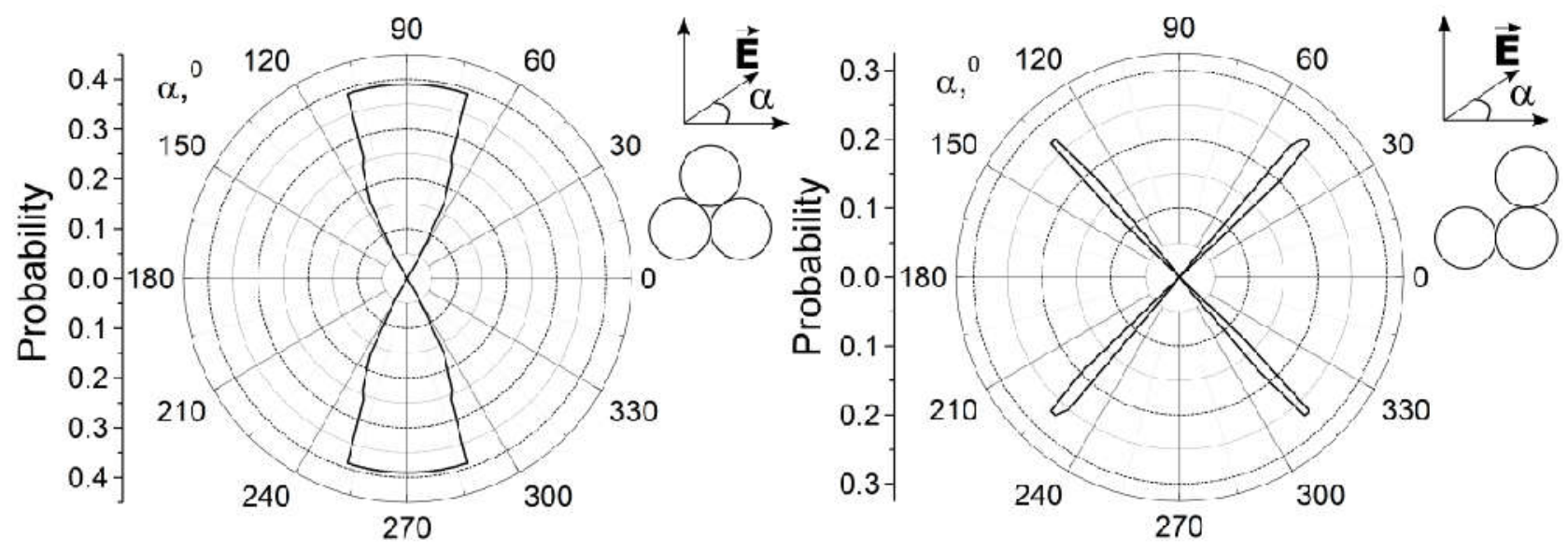

Fig. 5. Probability of structure formation from three particles with spatially oriented pair in dependence on their mutual orientation at $\lambda^{r}=450 \mathrm{~nm}$. 


\subsection{Formation of structure from three particles with the participation of arbitrarily oriented pair of particles}

In the second case we will assume that particles 1 and 2 are preliminarily assembled into stable pair that is arbitrarily oriented in space. Like in previous case, the task is the numerical calculation of energy of interaction between the third particle and pair of particles 1 and 2 in dependence on wavelength and external field polarization. Starting position of particles is the same as in Fig. 1. Dependence of dipole-dipole interaction energy normalized to room temperature thermal energy on the wavelength and angle $\alpha$ for three particles is shown in Fig. $6\left(r_{23}^{\frac{1}{2}}=16 \mathrm{~nm}\right)$ for $\theta=0^{\circ}(6 \mathrm{a})$, and $\theta=120^{\circ}(6 \mathrm{~b})$.

As one can see from Fig. 6, for $\theta=0^{\circ}$ at $\alpha=0^{\circ}$ and $\alpha=180^{\circ}$ (Fig.. 6a) the potential well in the interaction energy of particles is formed with the depth of order $40 \mathrm{kT}$ and spectral width $110 \mathrm{~nm}$. Different behavior is observed for $\theta=120^{\circ}$, where potential well with the depth $5.5 \mathrm{kT}$ and spectral width $70 \mathrm{~nm}$ is formed at $\alpha=90^{\circ}$ (Fig. 6b). Similarly to previous case, reduction of the distance between the pair and free particle leads to the increase of detuning off resonance, and the resonant wavelength becomes shifted to longer-wavelength (shorter wavelength) region of spectrum for $\theta=0^{\circ}\left(\theta=120^{\circ}\right)$, correspondingly (Fig. 7).

Longer-wavelength maximum of potential well is attained at the external field wavelength $\lambda^{r}=740 \mathrm{~nm}$. Probability of adjoining of free particle in external resonant field in dependence on its polarization during time period no more than $10 \mathrm{~ns}$ is presented in Fig. 8.

a)

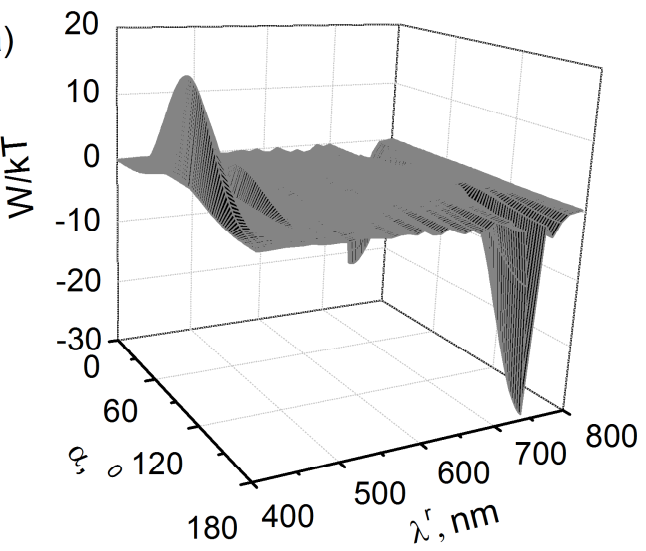

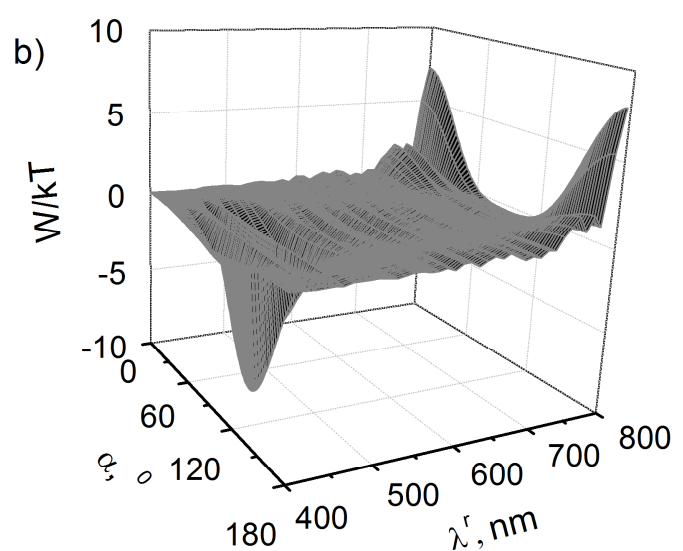

Fig. 6. Dependence of time-averaged dipole-dipole interaction energy of three particles with arbitrarily oriented pair on polarization angle and external field wavelength for $\theta=0^{\circ}(6 \mathrm{a}), \theta=120^{\circ}(6 \mathrm{~b})$. 

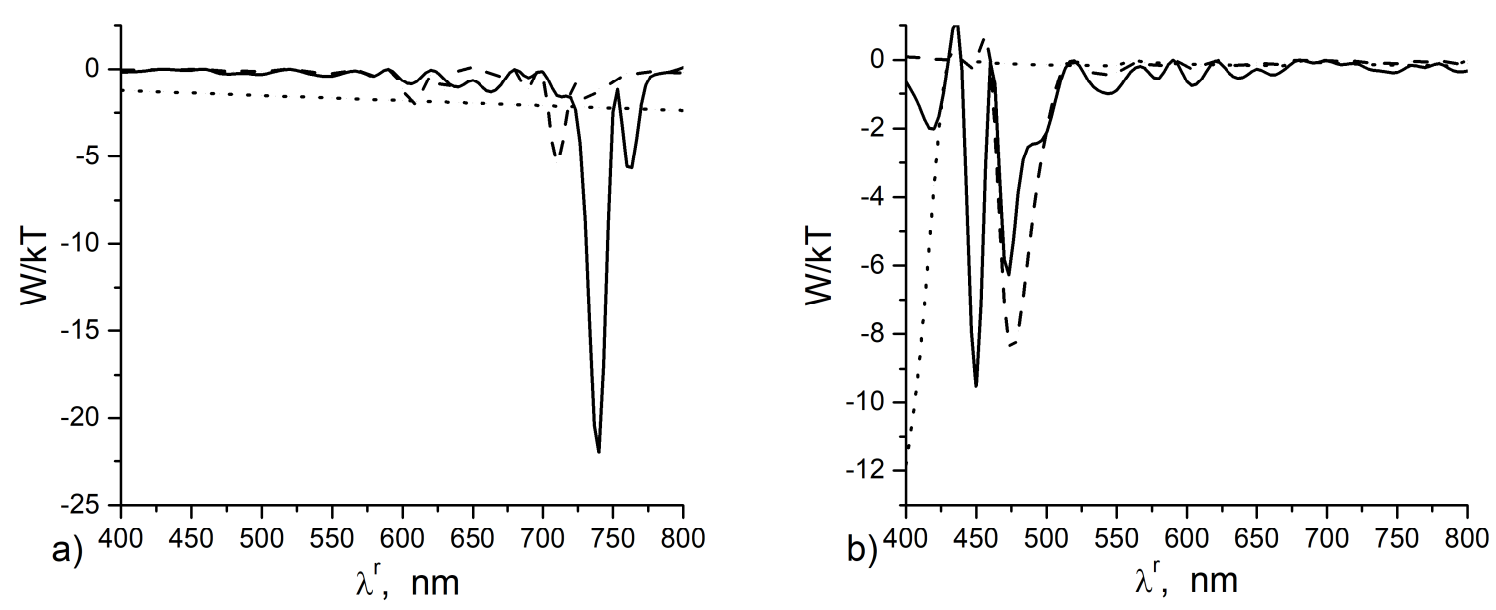

Fig. 7. Dipole-dipole interaction energy of three particles with arbitrarily oriented pair in dependence on interparticle distance for $\theta=0^{\circ}, \alpha=0^{\circ}(7 \mathrm{a})$ and $\theta=120^{\circ}, \alpha=90^{\circ}(7 \mathrm{~b})$. Dash lines correspond to interparticle distance $r_{23}=20 \mathrm{~nm}$, solid lines $-16 \mathrm{~nm}$, dot lines $10 \mathrm{~nm}$.

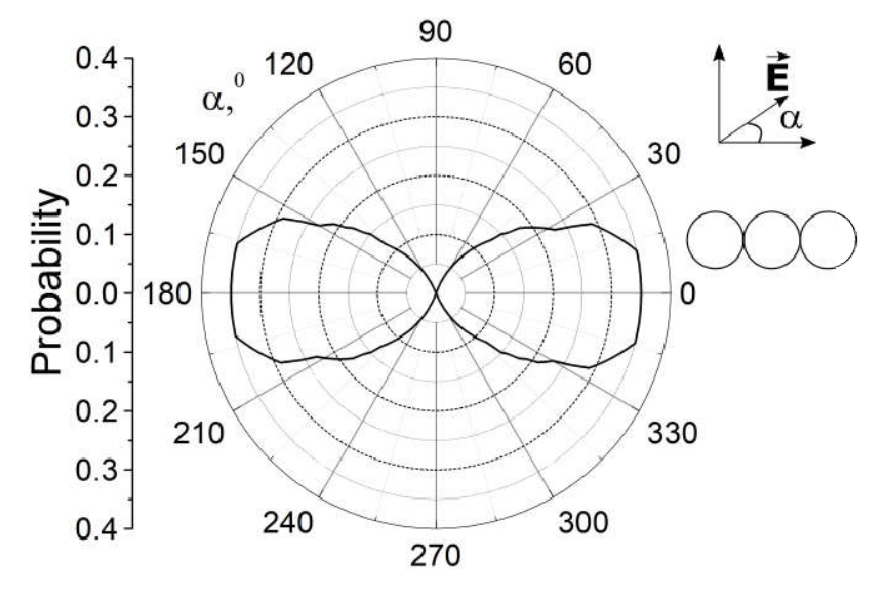

Fig. 8. Probability of structure formation from three particles with arbitrarily oriented pair in dependence on their mutual orientation at $\lambda^{r}=740 \mathrm{~nm}$.

According to calculation, for the quoted wavelength predominantly linear structures are formed. Average adjoining time is $7.45 \mathrm{~ns}$. Probability of required structure formation is $35 \%$, while $43 \%$ of runs do not resulted in formation of any structure, and $22 \%$ of runs terminated by formation of structure other than linear. Angle of external field polarization direction that is favorable for attaining maximum probability of linear structure formation equals to $0^{\circ}$. Oppositely, shorterwavelength potential well is preserved at $\lambda^{r}=450 \mathrm{~nm}$ (Fig. 9) and is most favorable for pyramid-type structure formation. 


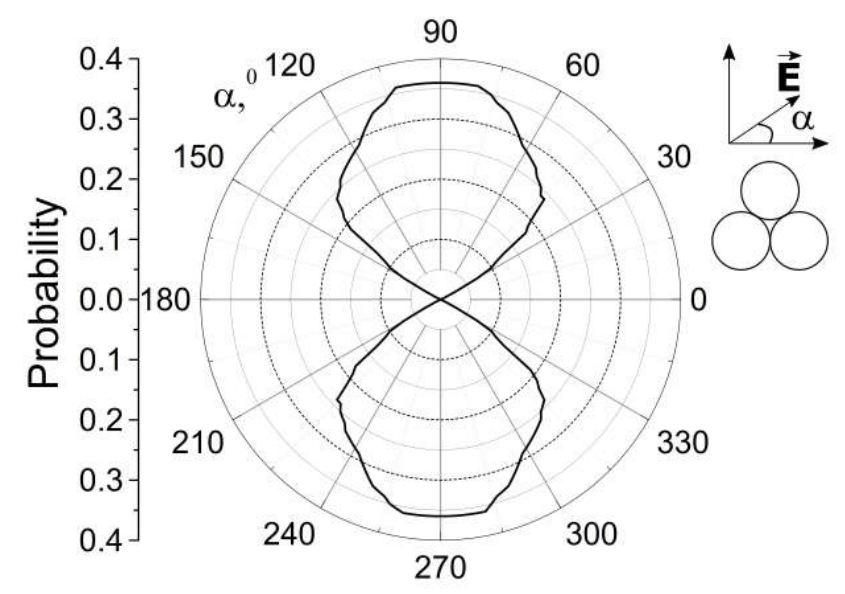

Fig. 9. Probability of adjoining of free particle to the arbitrarily oriented pair into pyramid-type structure in dependence on their mutual orientation at $\lambda^{r}=450 \mathrm{~nm}$.

Average pyramid-type adjoining time for $\lambda^{r}=450 \mathrm{~nm}$ is $4.89 \mathrm{~ns}$, and maximum probability of pyramid typestructure formation is $36 \%$ at external field polarization angle $90^{\circ} ; 33 \%$ of runs result in absence of adjoining while in the rest of runs the structure other than pyramid are formed.

Therefore, formation of three-particle structure via adjoining of the free particle to the preliminarily formed pair is possible without additional orienting field. Structure formation probabilities more than one third per single $10 \mathrm{~ns}$ pulse can be attained. Selectivity on the laser wavelength allows directed formation of predominantly either linear or pyramid-type structures.

The employment of realistic parameters of medium and the field, as well as good qualitative agreement between earlier calculations [21] and the experiment on the formation of pairs of colloidal quantum dots in the field of laser radiation [22] evidence the possibility of implementation of similar self-assembly of complex structure in the real experiment. This experiment can be done with the help of optical parametric oscillator at pulse duration of order $10 \mathrm{~ns}$ and the tuning range in the visible depending on the size of individual nanoparticles.

\section{Conclusion}

Present study has shown that, for the example of three-particle structure, the formation of complex structures can be achieved in the course of step-by-step process when the third particle is adjoining to a preliminarily formed pair. Initial pair in this case can be either fixed by auxiliary field or arbitrarily oriented in space. The first approach allows achieving larger structure formation probability ( $46 \%$ per a single pulse) and obtaining three different geometries of resultant structures. This approach uses two independently controllable parameters of laser radiation, namely, wavelength and orientation of electric field strength vector. In case of second approach, the structure formation probability per pulse is smaller (36\%), and formation of only two kind of structures is possible. However, the probabilities of formation of stable structures in both approaches do not differ strongly, and the second approach do not require additional orienting field. The latter means that second approach is easier to be implemented in the experiment.

The laser source required for pre-defined structures formation must be widely tunable across the visible and must generate pulses with the duration of order of $10 \mathrm{~ns}$. These requirements are well fitted by commercial optical parametric oscillators, and consequently, the proposed technique is well implementable in practice.

\section{Acknowledgements}

The reported study was funded by Russian Science Foundation (Grant 18-72-00003). V.V. Slabko is grateful for the support from the Ministry of Education and Science of the Russian Federation (Grant 3.6341.2017/VU).

\section{References}


[1] A.S. Arico, P. Bruce, B. Scrosati, J.M. Tarascon, W. Van Schalkwijk, Nanostructured materials for advanced energy conversion and storage devices, Nat. Mater. 4(5) (2005) 366-377. doi:\{10.1038/nmat1368\}.

[2] C.L. Chen, S.C. Chang, C.K. Chen, C.K. Chang, Lattice Boltzmann simulation for mixed convection of nanofluids in a square enclosure, Appl Math Model 39(8) (2015) 2436-2451. doi:\{10.1016/j.apm.2014.10.049\}.

[3] H. Caiyun, K. Xianggui, J. Meihong, L. Xiaodong, Metal Ni-decorated Fe3O4 nanoparticles: A new and efficient electrocatalyst for oxygen evolution reaction, J. Mater. Sci. 53(2) (2018) 1123-1134. doi:\{10.1016/j.matlet.2018.03.142\}.

[4] H. Mohsen, M. Mahdiye, S. Meisam, D. Franck, V. Henri, New polyvinyl chloride (PVC) nanocomposite consisting of aromatic polyamide and chitosan modified $\mathrm{ZnO}$ nanoparticles with enhanced thermal stability, low heat release rate and improved mechanical properties, Appl. Surf. Sci. 439 (2018) 1163-1179. doi:\{10.1016/j.apsusc.2018.01.255\}.

[5] A. Isiaka, N. H. Mahouton, Vector coherent states for nanoparticle systems, J. Phys. A: Math. Theor. 44(46) (2011) №465208. doi: $\{10.1088 / 1751-8113 / 44 / 46 / 465208\}$.

[6] D. S.P. Salazar, S. A. Lira, Exactly solvable nonequilibrium Langevin relaxation of a trapped nanoparticle, J. Phys. A: Math. Theor. 49(46) (2016) 1-17. doi: $\{10.1088 / 1751-8113 / 49 / 46 / 465001\}$.

[7] A. Peleš, O. Aleksić, V. P. Pavlović, V. Djoković, R. Dojcilović, Z. Nikolić, F. Marinković, M. Mitrić, V. Blagojević, B. Vlahović, V. B. Pavlović, Structural and electrical properties of ferroelectric poly(vinylidene fluoride) and mechanically activated $\mathrm{ZnO}$ nanoparticle composite films, Phys. Scr. 93(10) (2018). doi: $\{10.1088 / 1402-4896 / \mathrm{aad} 749\}$.

[8] A. Moradi, Collective excitations of spherical semiconductor nanoparticles, Phys. Scr. 91(10) (2016). doi: $\{10.1088 / 0031-8949 / 91 / 10 / 105802\}$.

[9] Y. Zhang, Z. Zhang, J. Guan, An empirical study of an agglomeration network, J. Phys. A: Math. Theor. 40(41) (2007)12365-12375. doi: $\{10.1088 / 1751-8113 / 40 / 41 / 007\}$.

[10] Y. Zhang, J. Tu, D. Wang, H. Zhu, S. K. Maity, X. Qu, B. Bogaert, H. Pei, H. Zhang, Programmable and multifunctional DNA-based materials for biomedical applications, Adv. Mater. 30(24) (2018). doi: $\{10.1002 /$ adma.201703658\}.

[11] G. Tikhomirov, S. Hoogland, P.E. Lee, A. Fischer, E.H. Sargent, S.O. Kelley, DNA-based programming of quantum dot valency, self-assembly and luminescence, Nat. Nanotechnol 6(8) (2011) 485-490. doi:\{10.1038/NNANO.2011.100\}.

[12] S.I. Stupp, Self-Assembly and biomaterials, Nano Lett. 10(12) (2010) 104783-4786. doi: \{10.1021/nl103567y\}.

[13] H.Yang, W. Cai, X. Guo, Preparation and infrared emissivities of self-assembled ZnO spherical aggregates, Mater. Sci. Semicond. Process. 24 (2014) 164-168. doi: $\{10.1016 /$ j.mssp.2014.03.030\}.

[14] J. Gong, G. Li, Z. Tang, Self-assembly of noble metal nanocrystals: Fabrication, optical property, and application, Nano Today 7(6) (2012) 564-585. doi:\{10.1016/j.nantod.2012.10.008\}.

[15] H. Liu, X. Zhang, Z. Gao, Lithography-free fabrication of large-area plasmonic nanostructures using colloidal gold nanoparticles, Photonics Nanostructures: Fundam. Appl. 8(3) (2010) 131-139. doi:\{10.1016/j.photonics.2010.02.002\}.

[16] D. Chu, K. Yin, D. Cui, X.Sun,Y. Hu, J.A. Duan, Nanostructures' difference for differing band gap materials during ultrashort double-pulse laser ablation, Photonics Nanostructures: Fundam. Appl. 33 (2019) 16-20. doi: $\{10.1016 /$ j.photonics.2018.11.003\}.

[17] K. Dholakia, P. Zemanek, Colloquium: Gripped by light: Optical binding, Rev. Mod. Phys.82 (2010) $1767-1791$. doi: $\{10.1103 /$ RevModPhys.82.1767\}.

[18] S.V. Karpov, A.K. Popov, V.V. Slabko, Photochromic reactions in silver nanocomposites with a fractal structure and their comparative characteristics, Tech. Phys. 48 (6) (2003) 749-756. doi: $\{10.1134 / 1.1583830\}$.

[19] V. V. Slabko, G. G. Khachatryan, and A. S. Aleksandrovsky, Self-organized aggregation of small metal particles controlled by an external light field, JETP Lett. 84(6) (2006) 300-304. doi:\{10.1134/S0021364006180056 \}.

[20] V. V.Slabko, A.S. Tsipotan, A.S. Aleksandrovsky, Resonant light-controlled self-assembly of ordered nanostructures, Photonics Nanostructures: Fundam. Appl. 10(4) (2012) 636-643. doi:\{10.1016/j.photonics.2012.06.002\}.

[21] V.V.Slabko, A.S. Tsipotan, A.S. Aleksandrovsky, E.A. Slyusareva, Dynamics of self-organized aggregation of resonant nanoparticles in a laser field, Appl. Phys. B 117(1) (2014) 271-278. doi:\{10.1007/s00340-014-5831-0\}.

[22] A.S. Tsipotan, M.A. Gerasimova, V.V. Slabko, A.S. Aleksandrovsky, Laser-induced wavelength-controlled selfassembly of colloidal quasi-resonant quantum dots, Opt. Express 24(10) (2016) 11145-11150. doi: \{10.1364/OE.24.011145\}. [23] S.V Karpov, I.L. Isaev, A.P. Gavrilyuk, General principles of the crystallization of nanostructured disperse, Colloid J. 71(3) (2009) 313-328. doi: $\{10.1134 /$ S1061933X09030053\}.

[24] L. D. Landau, E. M. Lifshitz, The Classical Theory of Fields, Butterworth-Heinemann, 1980.

[25] F. Hafezi, R.S. Ransing, Computational modelling of fluid structure interaction at nanoscale boundaries with modified Maxwellian velocity distribution, Appl. Math. Model. 37(14-15) (2013) 7504-7520. doi: \{10.1016/j.apm.2013.04.013\}.

[26] Ch. Kittel Introduction to Solid State Physics, 8th edition, Wiley, 2004. 
[27] J.C. Butcher, Numerical Methods for Ordinary Differential Equations, John Wiley \& Sons, New York, 2008. 